\title{
Pediatric Obesity: Influence on Drug Dosing and Therapeutics
}

\author{
Barbara Ameer PharmD, MBA, BCPS, FCP \\ Rutgers - Robert Wood Johnson Medical School \\ Michael Weintraub MD \\ Thomas Jefferson University
}

Follow this and additional works at: https://jdc.jefferson.edu/medfp

Part of the Medical Pharmacology Commons, Pediatrics Commons, and the Pharmacy and Pharmaceutical Sciences Commons

Let us know how access to this document benefits you

\section{Recommended Citation}

Ameer, Barbara PharmD, MBA, BCPS, FCP and Weintraub, Michael MD, "Pediatric Obesity: Influence on Drug Dosing and Therapeutics" (2018). Department of Medicine Faculty Papers. Paper 228.

https://jdc.jefferson.edu/medfp/228

This Article is brought to you for free and open access by the Jefferson Digital Commons. The Jefferson Digital Commons is a service of Thomas Jefferson University's Center for Teaching and Learning (CTL). The Commons is a showcase for Jefferson books and journals, peer-reviewed scholarly publications, unique historical collections from the University archives, and teaching tools. The Jefferson Digital Commons allows researchers and interested readers anywhere in the world to learn about and keep up to date with Jefferson scholarship. This article has been accepted for inclusion in Department of Medicine Faculty Papers by an authorized administrator of the Jefferson Digital Commons. For more information, please contact: JeffersonDigitalCommons@jefferson.edu. 
Title: Pediatric Obesity: Influence on Drug Dosing and Therapeutics

\author{
Authors and Affiliations: \\ Barbara Ameer, PharmD, MBA, BCPS, FCP \\ Department of Medicine, Rutgers - Robert Wood Johnson Medical School, \\ Piscataway NJ 08854
}

Michael A. Weintraub, MD

Department of Medicine, Thomas Jefferson University Hospitals, Philadelphia PA 19107-5084

\title{
Corresponding Author:
}

Dr. Barbara Ameer, P O Box 818, Princeton Jct, NJ 08550-0818

(ameerbcps@gmail.com)

Dr. Ameer is a Fellow of the American College of Clinical Pharmacology (FCP)

Disclosures: The authors have no conflicts relevant to this work.

Word count for body of manuscript: 6,369

Total number of figures and tables: 6 (2 figures including 1 color figure, 4 tables)

and 1 supplemental table

References: 94 


\begin{abstract}
Obesity is an ongoing global health concern and has only recently been recognized as a chronic disease of energy homeostasis and fuel partitioning. Obesity afflicts $17 \%$ of US children and adolescents. Severe obesity $\left(\geq 120 \%\right.$ of the $95^{\text {th }}$ percentile of BMI-for-age, or a BMI of $\geq 35 \mathrm{~kg} / \mathrm{m}^{2}$ ) is the fastest growing subgroup and now approaches $6 \%$ of all US youth. Health consequences (e.g., type 2 diabetes, coronary heart disease) are related in a dose-dependent manner to severity of obesity. Since therapeutic interventions are less effective in severe obesity, prevention is a high priority. Treatment plans involving combinations of behavioral therapy, nutrition and exercise achieve limited success. Only one drug, orlistat, is FDA-approved for long-term obesity management in adolescents 12 years and older. As part of comprehensive medication management, clinicians should consider the propensity for a given drug to aggravate weight gain and to consider alternatives that minimize weight impact. Medication management must take into account developmental changes as well as pathophysiology of obesity and comorbidities.

Despite expanding insight into obesity pathophysiology, there are gaps in its translation to therapeutic application. The historical construct of obesity as simply a fat storage disorder is fundamentally inaccurate.

The approach to adjusting doses based solely on body size and extrapolating from therapeutic knowledge of adult obesity may be based on assumptions that are not fully substantiated. Classes of drugs commonly prescribed for comorbidities associated with obesity should be prioritized for clinical research evaluations aimed at optimizing dosing regimens in pediatric obesity.
\end{abstract}

Keywords Adolescent medicine, Obesity, Pediatrics, Type 2 diabetes, Therapeutics, Comprehensive medication management 


\section{Introduction}

Pediatric obesity with its metabolic complications is a public health problem recognized as a major contributor to health burden on a global scale. Clinical data supports the American Medical Association's decision in 2013 to designate obesity as a disease. ${ }^{1}$ The obesity epidemic has led to the emergence among adolescents of what have previously been predominantly adult-onset diseases: type 2 diabetes, hypertension, nonalcoholic liver disease, dyslipidemia and obstructive sleep apnea. ${ }^{2}$ Direct medical costs for provision of care to obese patients in the United States was estimated at $\$ 147$ billion per year in $2008 .{ }^{3}$ Such estimates of incremental cost of care do not include diminished quality of life for patients and their families, which is part of the cost of obesity with its attendant morbidities.

Considering that cases are projected to increase over time, the future costs to society are staggering. Many factors must be considered in planning how best to apportion health care resources, how to determine clinical priorities and treatment approaches, how to prioritize clinical research, and how to advance drug development for more and better treatment options. 


\section{Definitions of Obesity in Children and Adolescents}

While it is an imperfect measure of body fat, body mass index (BMI) correlates fairly well with percentile rankings of percent body fat when it is measured by more direct methods. ${ }^{4} \mathrm{BMI}$ is the most commonly used clinical screening measure of overweight and obesity, followed by waist circumference and waist-to-hip ratio as clinical measures of intra-abdominal adipose tissue accumulation, called visceral adiposity. In extremely obese youth, waist circumference is not recommended because it is less accurate in this subgroup. ${ }^{4}$

BMI growth charts are tools for tracking changes in overweight and obesity status among children aged $\geq 2$ years. Since a child grows in weight as well as height, the norms for BMI (the quotient of weight in kilograms and the square of height in meters, expressed as $\mathrm{kg} / \mathrm{m}^{2}$ ) vary with age and sex, as seen in the charts and online calculator from the Centers for Disease Control and Prevention (CDC). ${ }^{5,6}$

Children aged $\geq 2$ years are diagnosed as obese if the BMI is $\geq 95^{\text {th }}$ percentile for ageand sex-based on the CDC growth charts published in May 2000, which is the most current. A diagnosis of overweight is made if $\mathrm{BMI}$ is $\geq 85^{\text {th }}$ percentile but $<95^{\text {th }}$ percentile for age and sex. ${ }^{7}$ For a child $<2$ years, obesity is diagnosed when the weight for recumbent length is $\geq 97.7^{\text {th }}$ percentile of the growth standards of the World Health Organization $(\mathrm{WHO})^{8}$ 
Severe obesity, also known as extreme obesity, is defined as either a BMI-for-age $\geq 120 \%$ of the $95^{\text {th }}$ percentile or a BMI of $35 \mathrm{~kg} / \mathrm{m}^{2}$. These criteria correspond to the $99^{\text {th }}$ percentile. Some clinician-researchers further stratify this subgroup of adolescents and young adults with high BMI to better predict risk for developing metabolic abnormalities, i.e., insulin resistance (IR) and altered glucose metabolism, low high-density lipoprotein (HDL) cholesterol and high systolic blood pressure. ${ }^{4}$ Using this approach, severe obesity is categorized as class 2 obesity, while class 3 is a BMl-for-age $\geq 140 \%$ of the $95^{\text {th }}$ percentile or a BMI of $40 \mathrm{~kg} / \mathrm{m}^{2}$.

This upward shift in BMI is a concern for the health of populations both now and far into the future. ${ }^{9}$ Higher levels of obesity carry the greatest cardiometabolic risk and potential for serious disease, complications and disability into adulthood. Furthermore, responses to treatment approaches are less effective in severe obesity. ${ }^{10}$

\section{Prevalence and Epidemiology}

As of 2014 , obesity afflicts $17.2 \%$ of all US children and adolescents, aged 2 to 19 years old, and another $16.2 \%$ are overweight. ${ }^{11}$ Taken together, overweight and obese individuals encompass about one-third of youth in the United States, ${ }^{12}$ as well as in the United Kingdom. ${ }^{13}$

US statistics on trends in childhood obesity utilize measured height and weight data obtained by the National Health and Nutrition Examination Survey (NHANES) over 
many decades. Based on data collected through 2013-2014, the most recent update, obesity either slowed its pace of rise or plateaued in certain age subgroups (Figure 1), but the durability of this trend remains to be seen.

As shown in Figure 2, there are racial/ethnic differences and sex differences in obesity occurrence in the US pediatric population. Notable are the low occurrence among Asian girls and the relatively high prevalence among Hispanics and Mexican Americans of both sexes.

More than half of the world's obese youth reside in countries with low- to middle-income economies. Statistics from these countries are sparse and may not be directly comparable to those generated in the United States if they use the child growth standards of the World Health Organization or national standards, rather than those of the CDC. In 2012, 37\% of school-aged boys in Mexico were overweight or obese. ${ }^{14}$ Latin American countries face a dual challenge of pediatric obesity and undernutrition. Through improved nutrition and daily physical activity programs at school, the Pan American Health Organization emphasizes prevention and weight management. ${ }^{14}$

With a continuously upward trend in its prevalence, severe obesity is the fastest growing subgroup. ${ }^{4}$ In NHANES data collected from 2011 to 2014 , severe obesity afflicted $5.8 \%$ of US youth overall. ${ }^{12}$ Among ethnic/racial subgroups, severe obesity was highest among non-Hispanic blacks at $8.6 \%$, followed by Hispanics at $7.6 \%$. For other non- 
Hispanic pediatric groups, the prevalence was $4.4 \%$ among white youth, and lowest among Asians at $1.3 \% .^{12}$

It is important to note that the correct interpretation of BMl among ethnic minorities is based upon ethnic norms. There are pediatric racial/ethnic differences in the percentage of body fat at a given BMI. With their body composition and pattern of lipid partitioning, Asians begin to develop cardiovascular risk at lower BMI thresholds. ${ }^{7,15}$ Using standard BMI cut points likely underestimates health risk among pediatric Asians.

As another means of describing unhealthy body composition, there are individuals with excess body fat even though their BMI might be normal, i.e., not overweight or obese. As was reported in one world region examined in 2008 in the CASPIAN study, this body composition described as many as $25 \%$ of adults. ${ }^{16,7}$

A recent public health report defined an overfat phenotype as excess body fat that can impair health, at any BMI. The umbrella term overfat encompasses not only overweight and obese but also "metabolically obese, normal weight" (MONW) such as visceral obesity. It affects about half of children residing in 30 developed countries ${ }^{17}$ and as many as $69 \%$ of youth ( 2 to 19 years) in the United States. ${ }^{18}$

Because excess body fat is associated with increased cardiometabolic risk, ${ }^{18} \mathrm{MONW}$ might be a factor contributing to heterogeneity among normal body weight patients recruited into metabolic research studies or in patient care settings, particularly among 
ethnic populations known to have low BMI values. ${ }^{19}$ Again, it is important to note the limitations of interpreting BMI similarly across all ethnic groups.

While there is no standardized definition, the obesity phenotype described as "metabolically healthy obesity" (MHO) is described as obesity with high insulin sensitivity and without diabetes, dyslipidemia or hypertension. MHO prevalence, which varies with the criteria applied, ${ }^{20}$ ranges from 20 to $30 \%$ of obese individuals of any age. ${ }^{21}$ Importantly, MHO may represent a step toward metabolically unhealthy obesity, ${ }^{22,23}$ at which point there is evidence of biochemical and metabolic changes. Whether drug disposition might be different in metabolically healthy obesity, as an early stage of the disease in youth or adults, compared to later stages of obesity is worth conjecture and scientific inquiry.

\section{Obesity Pathogenesis}

Obesity was once regarded simply as a storage disorder of adipose tissue. It is now described as a complex disorder of energy balance that involves adipocytes, the gastrointestinal tract, and the brain, through its operational control of food intake and energy expenditure. With ample evidence that the disorder has many features of a chronic systemic disease,${ }^{1}$ there is much ongoing research into the gut-brain axis, neurobiology determinants of body fat mass, as well as the interplay of genetics, epigenetics, developmental influences and the environment. There rarely is a single genetic cause of an underlying hormonal abnormality that leads to early weight gain in 
infants. A discussion of these topics is beyond the scope of this paper, but can be found in a recent review. ${ }^{24}$ What follows is a brief overview of pathophysiology of obesity to support an understanding of pharmacotherapy.

In overweight or obese individuals, there is a sustained positive energy balance (energy intake exceeding energy expenditure) combined with an inherited predisposition for weight gain. A related but distinct process is that body weight is maintained at a stable range, referred to as the set point, regardless of variation in energy intake and expenditure. The control mechanism is located in the lateral hypothalamus. The body has evolved to be efficient at protecting against weight loss during food deprivation or low intake. Weight that is gained is biologically defended, through biologic mechanisms that are still being elucidated. The higher weight then reestablishes the individual's set point at an elevated value.

Bariatric surgery, e.g., Roux-en-Y gastric bypass (RYGB), appears to lower the set point, accompanied by weight loss and high rates of remission of type 2 diabetes. ${ }^{25}$ Surgery has potential side effects, however, and is not a feasible option for many adolescents since it is only considered in mature teenagers who have access to skilled surgical centers and insurance coverage. These facts should reinforce the importance of slowing the rate of weight gain or avoiding weight gain during adolescence by lifestyle interventions, i.e., physical activity and nutritional meal planning, along with pharmacotherapy. 
Several key organs and peptide hormones are involved in human energy homeostasis. ${ }^{26}$ The peptide ghrelin is orexigenic, as it signals from the stomach to the brain to stimulate appetite. Insulin, on the other hand, signals the brain to curtail feeding. This is in addition to insulin's actions that promote nutrient uptake into muscle, liver and fat.

In addition to fatty acids, adipose tissue is an endocrine organ that produces hormones. ${ }^{27}$ The adipocyte hormone leptin circulates in concentrations that are proportional to body fat mass. ${ }^{28}$ It is believed that leptin evolved to be a metabolic switch that controls fat retention during periods of famine. ${ }^{27}$ Intact leptin-regulated neuronal circuitry is required for body weight and energy homeostasis. During and following loss of weight, there is a decline in leptin blood concentrations, which alters the brain's executive function and reward aspects of eating behavior. As a consequence, there is both a stimulation of feeding and a reduction in energy expenditure. Together these processes defend a higher level of body fat and serve to restore body weight to its original set point. ${ }^{24}$ This physiologic response to weight loss likely explains the regain of weight following initial successful weight loss from most forms of obesity treatment.

It seems particularly advantageous to avoid large weight gains prior to the time when the set point is established, since the highest body weight will be physiologically defended. It is unclear, however, when the set point is fixed but it is thought to happen sometime before adulthood, i.e., later adolescence ${ }^{29}$ Knowing this timing might help 
clinicians determine when to initiate pharmacotherapy for weight loss/management and to intensify efforts to achieve individualized body weight goals.

Leptin has a direct influence on lipid partitioning into skeletal muscle and adipocytes. It is hypothesized that the relative proportion of lipids in fat deposits, rather than the absolute amount, is the main determinant of metabolic risk. Different patterns of lipid partitioning may explain why some obese individuals develop comorbidities and others do not. ${ }^{24}$

Currently no pharmacologic therapy is known to lower the weight set point. Although recombinant human leptin analog, a marketed product for leptin deficiency and generalized lipodystrophy, is contraindicated in general obesity, it was experimentally shown to prevent the regain of weight in previously obese individuals who lost weight. ${ }^{30,31}$ This finding adds credence to the theory that a pharmacologic agent could be developed to reset the defended body weight in obese people to a value that is closer to normal. ${ }^{32,24}$

Other molecules (i.e., gut peptides, neurotransmitters, cytokines, steroid hormones, enzymes) play a role in human energy homeostasis. ${ }^{27}$ As underlying mechanisms are elucidated, it is expected to have a positive impact on developing new therapies to regulate body weight. 


\section{Clinical Aspects: Comorbid Conditions}

Obesity is accompanied by significant health problems. Most obese children continue to be obese as adults. Even early in childhood, weight-related morbidities can emerge. Childhood obesity is a risk factor for hypertension, type 2 diabetes, metabolic syndrome, and liver disease during adolescence, as well as for premature death in adulthood.

Pediatric clinical practice guidelines advise that children or adolescents with a BMI $\geq$ $85^{\text {th }}$ percentile be clinically evaluated for potential comorbidities, especially altered glucose metabolism with whole-body reduced insulin sensitivity, and fatty infiltration of the liver, known as nonalcoholic fatty liver disease (NAFLD). ${ }^{7}$ These conditions are among the first obesity-related comorbidities to emerge in childhood. Clinical manifestations of cardiometabolic risk and atherogenesis might develop sooner in boys than in girls, in whom high post-pubertal estrogen levels lower cardiovascular risk. ${ }^{10}$

The clustering of cardiometabolic risk factors, referred to as metabolic syndrome, is associated with increased BMI across different ethnic and racial groups of severely obese youth. Severe obesity is accompanied by a marked elevation of leptin that indicates abnormal satiety signaling and signifies future weight gain over time. ${ }^{4}$

When severe obesity has its onset during childhood, it portends a high level of cardiovascular and metabolic risk and a high prevalence of type 2 diabetes. Since severe obesity was uncommon decades ago, longitudinal data on health outcomes are 
still accruing. It is too soon to know the extent to which health outcomes will be more grave and costly in severely obese youth (class 2 or 3, see Definitions of Obesity) compared to children with normal body weight or even to those with class 1 obesity.

In addition to cardiovascular disease and diabetes, obesity is associated with numerous other conditions or risk factors (Table 1). ${ }^{15}$ Prominent among these are hepatic and gastrointestinal diseases. ${ }^{33}$

Obesity is considered a direct cause of NAFLD. ${ }^{34}$ Associated with both visceral adiposity and insulin resistance, NAFLD is the most common type of pediatric liver disease.$^{2}$ NAFLD is a spectrum of liver disease that necessitates attention to dosage adjustments as may be appropriate for certain hepatically-cleared drugs. ${ }^{35}$ In its most severe form, NAFLD can lead to cirrhosis and even liver transplant in adolescents.

Obesity is a risk factor for reflux esophagitis and gallstones. These gastrointestinal disorders might present at an earlier age or as a more complicated disease than in the absence of excess weight.

Additional conditions associated with obesity include obstructive sleep apnea, depression and other psychiatric conditions and disordered eating (i.e., binge eating and loss-of-control eating). ${ }^{36}$ Negative effects of weight gain include an increased cancer risk with some cancers having an onset in early adulthood. ${ }^{37}$ It is not well- 
appreciated by the public that excess body fat leads to greater risk for at least 13 different types of invasive cancers (e.g., colon, kidney, thyroid and pancreas). ${ }^{38}$

Most obesity-associated health conditions are chronic diseases that eventually require drug treatment. More studies are needed in the pediatric population to safely and effectively manage these common comorbidities with pharmacotherapy.

\section{Clinical Aspects of Medication Use: Medications Affecting Body Weight}

\section{Introduction}

Some drugs are associated with an undesired gain in body weight (Table 2), which can aggravate the underlying medical condition being managed with pharmacotherapy and lead to medication nonadherence ${ }^{39}$ If the agent is taken on an ongoing basis, weight gain is cumulative.

A medication management review of obese pediatric patients can help identify current or planned medications with a propensity to exacerbate weight gain. This section highlights several therapeutic classes of interest to this population, along with some alternative drugs with no known obesogenic effects in youth. It should be noted that some alternative drugs might be prescribed in youth despite being off-label for pediatric use based on current FDA-approved prescribing information. ${ }^{40}$

\section{Glucocorticoids}


With 60 years of clinical use, it is widely known that glucocorticoids impact body weight and fat distribution. Glucocorticoids stimulate appetite, despite elevations in leptin, and influence food preference towards protein and carbohydrates which lead to increased energy intake in excess of energy expenditure. ${ }^{41}$ Glucocorticoid-induced weight gain and lipodystrophy also involve effects on central and peripheral adipose tissue. ${ }^{42}$ Since glucocorticoid receptor density is higher in visceral adipose tissue relative to other fat depots, glucocorticoid-induced weight gain is typified by central (visceral) fat accumulation but with possible thinning of subcutaneous fat. This pattern of lipodystrophy is related to glucocorticoid effects on peripheral fat, where lipolysis is increased and lipoprotein lipase activity is decreased. ${ }^{42}$ The net effect is adipose tissue expansion as well as fat redistribution.

Weight gain is most notable at prednisone daily doses of $\geq 5 \mathrm{mg} .{ }^{43}$ The lowest possible dose is advisable in pediatric patients to minimize negative effects on linear growth and development as well as body weight. ${ }^{44}$ Depending on the indication, the substitution or addition of another effective, non-steroidal drug may provide a steroid-sparing effect.

\section{Diabetes Medications}

Several anti-diabetes drugs cause weight gain, which intensifies cardiovascular risk factors accompanying diabetes. Insulin is associated with weight gain, although the magnitude of effect varies with the intensity of the overall diabetes treatment regimen and with the type of insulin. 
In two randomized trials in pediatric patients with type 1 diabetes, insulin detemir had a more favorable effect on body weight than NPH insulin. ${ }^{45,46} \mathrm{BMI}$ was standardized (i.e., corrected for growth) to compare across the age range of 347 youth (6 to 17 years) in one six-month study ${ }^{45}$ as well as in 347 children ( 2 to 16 years) in a one-year trial. ${ }^{46} \mathrm{At}$ the end of both trials, weight ${ }^{46}$ or standard deviation weight scores ${ }^{45}$ were increased with NPH insulin but slightly decreased with insulin detemir.

Adult data support this finding for insulin detemir relative to NPH and basal insulin analogs at similar levels of glycemic control and hypoglycemia risk. In overweight adults with type 1 diabetes, NPH insulin was associated with a weight gain of $1.7 \pm 2.46 \mathrm{~kg}$, whereas insulin detemir exhibited slight weight reduction $(0.7 \pm 1.85 \mathrm{~kg})$ after 16 weeks. ${ }^{47}$ The physiological basis of this intrinsic property of insulin detemir is primarily reduced caloric intake (unrelated to hypoglycemia frequency), with the brain playing a key role in mediating the reduction of energy intake. ${ }^{47,48}$

The differing effects among insulin analogs provide caution against judging drugassociated weight gain as a pharmacologic effect common to all drugs in a given class. Each drug should be considered individually and monitored for body weight change during chronic dosing.

In pediatric diabetes management, it is common to initiate metformin. Metformin is less effective in youth-onset compared to adult-onset type 2 diabetes. ${ }^{49}$ Nearly half of youth 
with new-onset type 2 diabetes do not maintain adequate glycemic control with metformin monotherapy, with a median time to failure of about 11 months. ${ }^{50}$

Combinations of drugs acting by different mechanisms and including one or more weight-sparing or weight-losing drugs may be tried. This approach may allow a lower dosage of the diabetes drugs contributing to weight gain, which in pediatric patients might be a sulfonylurea (e.g., glimepiride, glyburide, glipizide), injectable insulin, and

possibly a thiazolidinedione (e.g., rosiglitazone), which was studied in the TODAY trial. ${ }^{39}$ Newer anti-diabetes agents with favorable effects on body weight include glucagon-like peptide-1 (GLP-1) receptor agonists, such as liraglutide and lixisenatide pen injector devices. ${ }^{51}$

Ongoing pediatric trials using adult anti-diabetes pharmacotherapy may lead to more choices and perhaps a labeled indication for treating diabetes without aggravating weight control. More and better options are particularly needed among young patients with diabetes and severe obesity.

\section{Antipsychotics, Second Generation}

Antipsychotic (AP) drugs are effective across a wide span of pediatric ages for the indications of autism spectrum disorder, schizophrenia, bipolar disorder, aggression and tics. ${ }^{52}$ 
With the advent of atypical or second-generation antipsychotics (SGAs), e.g., risperidone, olanzapine, quetiapine, introduced in the late 1990s, APs were heralded for less motor effects than traditional neuroleptics. That favorable safety feature was offset, however, by enduring negative effects on body weight, insulin resistance and lipid levels. ${ }^{53}$

The long-term impact of SGAs on weight along with metabolic and cardiovascular consequences, when instituted early in life, is now being realized as a generation of youth with psychiatric illness has been exposed to them for about two decades. ${ }^{54}$

Weight gain is evident soon after initiating antipsychotic therapy. Among 272 treatmentnaïve youth (from 4 to 19 years) initiated on SGAs, substantial weight gain was evident during the first 3 months of treatment. ${ }^{55}$ Weight gain was highest with olanzapine (mean $+8.5 \mathrm{~kg}, 95 \% \mathrm{Cl} 4.9$ to 7.2 ) and lowest with aripiprazole (mean $+4.4 \mathrm{~kg}, 95 \% \mathrm{Cl} 3.7$ to 5.2), with quetiapine- and risperidone-treated youth showing intermediate effects (mean $+6.1 \mathrm{~kg}$ and $+5.3 \mathrm{~kg}$, respectively).

Furthermore, over half of the patients ${ }^{55}$ experienced a clinically important change in body weight, defined as $>7 \%$ increase compared to baseline within 3 months of starting SGA monotherapy. ${ }^{56}$ An individual's vulnerability to significant weight gain is likely a combination of genetics, neurotransmitters, hormones, neuropeptides and lifestyle behaviors.R After accounting for weight gain expected in growing youth, the magnitude of weight gain with SGAs is greater in children and adolescents compared to adults. ${ }^{57,56}$ 
Adding metformin may slow the rate of weight gain or even decrease body weight during maintenance treatment. In a multi-center study of 61 autism spectrum disorder patients who experienced $>7 \%$ increase in body weight during treatment with SGAs, 29 youth were randomized to metformin liquid formulation, either 500 twice daily (for ages 6 to 9 years) or $850 \mathrm{mg}$ twice daily (10 to 17 years), and 32 to placebo. ${ }^{58}$ The primary study endpoint, the BMI standard deviation score (BMI z score), showed statistically greater reduction from baseline to week 16 among metformin-treated patients $(-0.10$, $95 \% \mathrm{Cl},-0.16--0.04, p=0.003) .{ }^{58}$ There were no significant effects on lipids, fasting insulin, fasting glucose, hemoglobin $\mathrm{A} 1 \mathrm{c}(\mathrm{HbA} 1 \mathrm{c})$ or other indicators of negative cardiometabolic consequences of SGA medication. It is speculated that the later findings were biochemical evidence of the cardiometabolic benefit of metformin offsetting SGA effects. ${ }^{58}$ Gastrointestinal side effects were common but well-tolerated, and not a reason for study discontinuation by 5 children in the metformin group.

Other SGAs with FDA-approved pediatric indications produce weight gain of varying degrees compared to placebo, as reported in their product label: asenapine, ${ }^{59}$ lurasidone,${ }^{60}$ and paliperidone.${ }^{61}$ Comparative trials with active control treatment arms are needed to understand the relative magnitude of weight gain with these newer SGAs.

\section{Antidepressants}


Antidepressants are increasingly prescribed for pediatric depression, anxiety disorders and obsessive-compulsive disorder. The selective serotonin reuptake inhibitors (SSRIs) citalopram and escitalopram were strongly associated with increase in BMI, visceral fat mass and visceral fat mass index in 264 older adolescents, aged15 to 20 years, who had not been medically treated within one month of initiating the antidepressant. The association with body composition was weaker for fluoxetine, whereas sertraline was no different than no SSRI treatment. ${ }^{62}$ Psychological therapy is an option that could be considered since it can achieve similar response rates as medication in children and adolescents with depression. ${ }^{63}$

\section{Antiepileptic Drugs}

Antiepileptic drugs have variable effects on body weight, based on reports from mixed age groups and not specifically pediatric populations. ${ }^{39}$ Sodium valproate (i.e., valproic acid), gabapentin, pregabalin and vigabatrin cause weight gain. ${ }^{64}$ Phenytoin, lamotrigine and levetiracetam are weight-neutral, whereas felbamate, topiramate and zonisamide can result in weight loss. ${ }^{64,65}$

\section{Clinical Aspects: Interventions For Control of Body Weight}

\section{Introduction}


Lifestyle interventions ideally are family-based efforts towards improving the patient's diet and lowering its caloric intake. These efforts are combined with increased physical activity and reduced sedentary behaviors. These lifestyle interventions are the foundation upon which drug treatment and surgery may be added. Bariatric surgery shows substantial improvement in weight loss and obesity-associated comorbidities. More knowledge is needed about the efficacy, safety and long-term consequences of bariatric surgery as well as long-term anti-obesity medication in a pediatric population.

Table 3 lists prescription drugs that have been and/or are currently in use in the United States for weight loss/management. This section highlights 3 classes of drugs - orlistat, metformin, and GLP-1 agonists - with existing or evolving long-term data on weight management effectiveness and safety in pediatrics. The mechanisms of action are mentioned under each class of medication.

Some of these drugs have been evaluated for beneficial impact on obesity-associated comorbidities. In multiyear-long studies in adults, the addition to lifestyle changes of either orlistat (Xenical in the Prevention of Diabetes in Obese Subjects, or XENDOS, a 4-year prospective study) ${ }^{66}$ or metformin (Diabetes Prevention Program Outcomes Study, or DPPOS, a 15-year extension of the landmark DPP trial) ${ }^{67}$ resulted in a delay in onset of type 2 diabetes in adults with prediabetes. Of the two agents, metformin has the stronger evidence base for effectiveness and safety for diabetes prevention. ${ }^{67} \mathrm{In}$ obese adolescents with IGT and a family history positive for type 2 diabetes, metformin 
improved glucose homeostasis and may prevent type 2 diabetes. ${ }^{68}$ There are no data on orlistat for diabetes prevention in the pediatric population. ${ }^{4}$

\section{Orlistat}

Currently orlistat is the only medication for long-term pediatric weight management approved, in December 2003, by US regulatory authorities. Orlistat is available both as a prescription (for children $\geq 12$ and adults) and a lower strength non-prescription product (for $\geq 18$ years).

In a clinical trial, 539 obese adolescents (with average BMl at the $98^{\text {th }}$ percentile) were randomized 2:1 to orlistat or placebo. The primary study endpoint of placebo-subtracted BMI change was $-0.86 \mathrm{~kg} / \mathrm{m}^{2}$ after one year of treatment, representing a BMI reduction of $2.4 \% .{ }^{69}$ Both active treatment and placebo groups had dietary instruction for caloric reduction accompanied by counseling for diet and exercise. A loss of at least $5 \%$ or $10 \%$ of body weight was achieved by $26.5 \%$ and $13.3 \%$, respectively, of the orlistat group and $15.7 \%$ and $4.5 \%$, respectively of the control group. The control group, however, did not maintain weight loss beyond 3 months. A $5 \%$ to $10 \%$ weight reduction is generally considered to produce beneficial effects on obesity-associated comorbidities, with improvement in their laboratory parameters.

To correct for poor absorption of certain dietary nutrients during orlistat, daily dietary supplementations that include fat-soluble vitamins are recommended several hours 
apart from orlistat, which is dosed with meals, three times a day. ${ }^{7}$ In a drug interaction study in normal weight young adults, orlistat did not significantly alter oral absorption of highly fat-soluble drugs fluoxetine or simvastatin that treat depression and dyslipidemia, respectively, which are not uncommon comorbidities in adolescent obesity (Table 1). ${ }^{70}$

Orlistat's gastrointestinal side effects, while not a significant issue reported in this research study, are common and poorly tolerated events related to orlistat's mechanism of action as a gastrointestinal lipase inhibitor and contribute to poor adherence. An analysis of orlistat prescription data in children and adolescents in the UK indicate a rate of discontinuation, for any reason, of $45 \%$ by the end of the first month and $75 \%$ by the third month. ${ }^{71}$

\section{Metformin}

Metformin has been used for many years for managing pediatric patients with overweight or obese individuals. ${ }^{4}$ Although its use for weight management is off-label, metformin is typically prescribed in youth for an on-label concurrent condition, which include polycystic ovary syndrome (PCOS), diabetes and prediabetes. A diagnosis of prediabetes is suspected when a $\mathrm{HbA} 1 \mathrm{c}$ value $\geq 5.7 \%$ is confirmed by impaired fasting plasma glucose (FPG: 100 to $125 \mathrm{mg} / \mathrm{dL}$ ) or an impaired glucose tolerance (IGT) (2hour plasma glucose: 140 to $199 \mathrm{mg} / \mathrm{dL}$ ). ${ }^{7}$ Since IGT exists in 10 to $30 \%$ of obese children and adolescents, ${ }^{15}$ many of these obese pediatric patients are eligible for metformin treatment for a labeled indication. 
Among 3 randomized controlled trials in which change in weight or BMI was the primary endpoint in obese youth 6 to 18 years old, metformin had a modest BMI effect. In these studies of 6-months ${ }^{72,73}$ or one-year duration, ${ }^{74}$ the placebo-subtracted change in $\mathrm{BMI}$ averaged $-1.1 \mathrm{~kg} / \mathrm{m}^{2}$ or approximately $3 \%$ reduction of BMI.

Differing responses to metformin as a weight-reducing medication may have multiple causes. Metformin tablets display low and variable absorption that could lead to variable drug effect. At physiologic $\mathrm{pH}$, metformin is a mono-protonated cation that relies upon active transport to cross lipid membranes, including those in the intestine, liver and kidney. Hepatic uptake is essential for metformin's full pharmacologic effect of inhibition of hepatic gluconeogenesis and increased insulin sensitivity. At the molecular level in the liver, metformin has an effect on the respiratory chain in the mitochondria. ${ }^{75}$ Metformin enters the liver, one of its sites of action, by several transporters, the predominant being the organic cation transporter 1 (OCT1), which exhibits polymorphisms. ${ }^{76}$

Genome-wide association studies indicate a genetic contribution to metformin's pharmacologic effects. Metformin disposition, pharmacodynamics and pharmacogenetics were retrospectively explored in 28 severely obese children. ${ }^{77}$ Polymorphisms in the gene SLC22A1 that expresses OCT1 resulted in lower levels of this protein transporter. A genetic variant might have negatively impacted metformin's efficacy of reducing adiposity in the subset of obese children who had insulin 
resistance. ${ }^{77}$ This hypothesis-generating retrospective analysis should be evaluated in a larger prospective comparative trial of obese and non-obese youth. A

pharmacogenomic approach might optimize dosing regimens and determine which drug transporter gene variants predict the patients who are unlikely to achieve clinically significant fat reduction or are likely to experience severe gastrointestinal side effects during metformin therapy. Furthermore, additional genetic studies of metformin might elucidate previously unidentified targets of its multiple molecular mechanisms.

\section{GLP-1 Agonists}

Exenatide was the first GLP-1 agonist to receive FDA approval, in 2005, for glycemic control in adults with type 2 diabetes. Although weight management is still not a labeled indication for any age group, this short-acting incretin mimetic produces weight loss in diabetic individuals, mainly or partly by decreasing appetite and slowing gastric emptying. In a pooled analysis of data from 32 adolescents 8 to 19 years of age with severe obesity enrolled in two small randomized trials, after 3 months of twice daily exenatide, the treatment effect on BMI relative to placebo was $-3.42 \%(95 \% \mathrm{Cl}:-5.41 \%$, $-1.42 \%){ }^{78}$ Predictors of this treatment effect were self-reported appetite at baseline and being female. Although not a predictor of response in the analysis, adherence with twice daily injections using a pen delivery device presents a challenge for this age group.

Liraglutide is the first GLP-1 mimetic to receive FDA approval, in 2014, for weight management with the indication restricted to adults. Liraglutide's weight loss efficacy 
and long-term safety at a once daily dose of $3 \mathrm{mg}$ are well-established in adults who are either overweight with comorbidities or are obese. ${ }^{79}$

Liraglutide doses up to $1.8 \mathrm{mg}$ have been studied for safety, pharmacokinetics (PK) and pharmacodynamics (PD) in a short-term trial of children and adolescents with type 2 diabetes but not obesity. Also, data from the obese pediatric population are accruing. These data include pharmacokinetic and safety findings from a recently completed trial in obese children aged 7 to 11 years, (NCT02696148) and a published safety, tolerability and PK study of 5-weeks duration, with dose escalation up to a maximum of $3 \mathrm{mg}$ in adolescents $\geq 12$ years (NCT01789086). ${ }^{80}$

Compared to oral metformin, high dose liraglutide in adults is associated with greater weight reduction, but GLP-1 mimetic injections are costly, less convenient and carry risk for serious but rare acute pancreatitis and acute gallbladder disease. With supporting clinical experience, GLP-1 agonist therapy may eventually become a welcomed pharmacologic option for weight control in youth with obesity.

\section{Other Agents: Topiramate and Phentermine}

The combination product topiramate/phentermine is indicated for obesity in adults but not children. Topiramate has not been studied in long-term randomized controlled trials in obese youth. Used in children as young as 2 years as an FDA-approved seizure medication, topiramate's observed anorexigenic and weight loss effects are attributed to 
the control of cravings. ${ }^{81}$ Concerning side effects limiting its usefulness in childhood obesity include paresthesias, cognitive disruption (confusion, difficulty concentrating) and teratogenicity (cleft palate).

Phentermine as a single agent is FDA-approved only in adolescents $>16$ years for short-term weight control ( $<3$ months at a time). This limited exposure achieves small to moderate weight loss. As a norepinephrine- and dopamine-releasing agent, phentermine may cause anxiety, tremors and slight increases in blood pressure.

\section{Non-Obesity Drugs Used in Hospitalized Pediatric Patients}

Table 4 lists commonly used medications in hospitalized pediatric patients and specifies whether the medicines have been specifically studied, prospectively or retrospectively, in obese individuals. The medication list is based on usage, which was collected in a drug interaction database accumulated from 42 U.S. pediatric intensive care units (PICUs) for patients ranging from infants, excluding neonates, to 17 years. ${ }^{82}$

Due to the paucity of published data on children and adolescents, the search for the listed drugs was also applied to studies in obese adults. The literature search was based on PubMed/Medline database searches October 11-22, 2017, with a publication date limit from 1950 to present and English language limit, using the following search terms and cross-references: "children" OR "child" or "adolescent" OR "adolescence" OR "youth" (included when searching for studies done in those $<18$ years old) AND "weight change" OR "weight gain" OR "overweight" OR "obesity" OR "obese" OR "BMI" OR 
"Body Mass Index" AND "drug dosing" OR "pharmacokinetics" AND "drug class" OR "specific drug name(s)." The search was augmented with a manual review of reference lists.

This exploratory literature evaluation focused on 7 therapeutic drug categories, excluding drugs that are mainly used in an acute care setting or in the context of surgical operations, e.g., propofol, and the neuromuscular blocking agents, rocuronium and vecuronium, but including antibiotics. Due to the pressing need to optimize drug dosing during, before and after bariatric surgery in recent years, drug research evaluations in the context of bariatric surgery have been conducted. A recent literature review discussed the following drugs relevant to obese adolescent surgical patients: acetaminophen, fentanyl and newer analogs, midazolam, enoxaparin, morphine, ketamine and dexmedetomidine. ${ }^{83}$

The most-used drugs from the PICU study are displayed as a list, ranked in order of prevalence of exposure, in Supplemental Table S1. More than half of the hospitalized pediatric patients had a prescription dispensed for acetaminophen, the single-most frequently ordered drug, with the following exposure rates by age category: infants: $57.9 \%$, children 1 through 9 years old: $58.0 \%$, adolescents 10 through 17 years old: $50.7 \%{ }^{82}$ Despite the high prevalence of exposure to acetaminophen, it has been the subject of very few obesity studies in adults ${ }^{84,85}$ or in children with the obesityassociated comorbidity of NAFLD. ${ }^{35}$ In the latter group, there is a concern that the cytochrome P450 2E1 pathway might be up-regulated in obesity, ${ }^{86}$ with the theoretical 
risk for increased formation of the hepatotoxic acetaminophen metabolite $\mathrm{N}$-acetyl-pbenzoquinone imine. Until research is conducted to elucidate metabolic pathways, it is prudent that, given their vulnerability for NAFLD, obese children and adolescents be monitored for aspartate aminotransferase and alanine aminotransferase elevations and other markers of liver function while on chronic acetaminophen at standard doses.

Antibiotics historically represent the largest portion of published clinical research on drugs in obesity, with several reviews covering that topic in pediatrics ${ }^{87,88}$ and adults. ${ }^{89,90}$ In the PICU study, antibiotics were collectively the most prevalent therapeutic category, with vancomycin as the most commonly prescribed antibiotic followed by various parenteral cephalosporins.

With the exception of antimicrobials, it is evident from this and other reviews of the literature that large gaps persist in our clinical knowledge about pharmacotherapy in pediatric obesity. ${ }^{91}$ In fact, a complete absence of pharmacokinetic information for obese children was noted by Rowe et al for 25 pediatric emergency care drugs. ${ }^{92}$

Where adequate studies exist in adult populations but not pediatrics, it is not uncommon to consider utilizing knowledge obtained from adult obesity studies to inform dosing recommendations. A main benefit is averting drug exposure to a vulnerable population while optimizing use of existing data. Spanning nearly 4 decades, however, obesity drug studies represent a wide range of research designs and reporting criteria. In the case of small studies of low quality, interpretation of the findings might be limited to hypothesis 
generation. Under certain circumstances, some drugs may deserve re-evaluation in well-designed controlled studies with application of modern technologies of sample and data analyses.

Extrapolation from existing pharmacokinetic or pharmacodynamic data from adults to the pediatric population, in general, has limitations. It is currently not known whether common obesity in an adult population is physiologically similar to obesity in childrenf or adolescents.

More knowledge is needed about the time course of adipocyte biology and pathophysiology and accompanying biochemical and metabolic changes to shed light on the validity of assumptions that may underlie extrapolation from other age groups with or without comorbidities.

In adults there are different phenotypes of obesity, including the metabolically healthy and unhealthy phenotypes, and subtypes with differences in the cardiometabolic risk profile and intracellular pattern of fat distribution. ${ }^{93}$ Furthermore, in some patients the disease changes over time. The progression of some overweight children to obesity during adolescence and then possibly severe obesity in later adolescence or into adulthood calls into question the likelihood that youth-onset obesity is equivalent to adult-onset obesity. 
A recent population pharmacokinetic modeling analysis of midazolam found that midazolam clearance in morbidly obese adults was not predictive of clearance in obese adolescents. ${ }^{94}$

Clinical studies typically do not collect and/or report duration of obesity, but this may be a variable of importance, for example, to compare similarity at baseline of demography in the treatment groups. More research is needed on drug transporters, drug metabolizing enzymes and their polymorphisms in both youth and adults, to better predict how drug disposition and metabolism are affected along the spectrum of obesity, compounded over time with accumulation of obesity comorbidities.

\section{Concluding Remarks}

Obesity is a heterogenous chronic disease of energy balance and fuel partitioning. About one-third of youth in the United States are overweight or obese and the subgroup of severe obesity is showing a persistently upward trend. For treating obesity in children and adolescents, pharmacotherapy options added to lifestyle modification are limited to orlistat, metformin for comorbidities which on on-label, and possibly off-label use of adult-approved antiobesity agents in selected patients. Obese youth with comorbidities will require medications as part of their chronic disease management, yet very few drugs have been studied in children or adolescents with obesity. This is an area of high need for study, since adult obesity may differ from obesity in youth and findings from 
pharmacokinetic investigations in obese adults may not predict pharmacokinetics in obese adolescents, as was shown with midazolam. Medication management should include judicious selection of drugs which will not hamper weight control. The highlights presented in this review of pediatric obesity pharmacotherapy should help prioritize areas of greatest need for further work.

\section{Acknowledgement}

The authors would like to acknowledge Donald Langan, Pharm.D., and M.D. candidate, for his assistance in conducting PubMed searches in support of Table 4. 


\section{Figure Legends}

Figure 1. The prevalence of obesity among youth in the United States shows an upward trend overall and by age subgroups of 2 to 5 years, 6 to 11 years, and 12 to 19 years. Data are from the Division of National Health and Nutrition Examination Surveys, reported through 2014. Pediatric definition of obesity is body mass index (BMI) $\geq 95$ th percentile using the sex-specific BMI-for-age 2000 Center for Disease Control (CDC) Growth Charts.

Figure 2. Dotted line indicates overall mean percent (17.2\%) of all US children and adolescents who are obese. Obesity prevalence data by ethnic/racial subgroups were obtained from the National Center for Health Statistics (NCHS), Division of National Health and Nutrition Examination Surveys, Health E-Stats, July 2016. Data from females exclude pregnant individuals. Pediatric definition of obesity is body mass index $(\mathrm{BMI}) \geq 95$ th percentile using the sex-specific BMI-for-age 2000 Center for Disease Control (CDC) Growth Charts. 


\section{References}

1. Hurt RT, Edakkanambeth Varayil J, Mundi MS, Martindale RG, Ebbert JO.

Designation of obesity as a disease: lessons learned from alcohol and tobacco. Curr Gastroenterol Rep. 2014;16(11):415.

2. Kumar S, Kelly AS. Review of Childhood Obesity: From Epidemiology, Etiology, and Comorbidities to Clinical Assessment and Treatment. Mayo Clinic proceedings. 2017;92(2):251-265.

3. Malik VS, Willett WC, Hu FB. Global obesity: trends, risk factors and policy implications. Nature reviews Endocrinology. 2013;9(1):13-27.

4. Kelly AS, Barlow SE, Rao G, et al. Severe obesity in children and adolescents: identification, associated health risks, and treatment approaches: a scientific statement from the American Heart Association. Circulation. 2013;128(15):16891712.

5. CDC. CDC Clinical Growth Charts. National Center for Health Statistics. Available at: https://www.cdc.gov/growthcharts/clinical charts.htm. Accessed September 26, 2017.

6. CDC. BMI Percentile Calculator for Child and Teen. Available at: https://nccd.cdc.gov/dnpabmi/Calculator.aspx. Accessed September 26, 2017.

7. Styne DM, Arslanian SA, Connor EL, et al. Pediatric Obesity-Assessment, Treatment, and Prevention: An Endocrine Society Clinical Practice Guideline. J Clin Endocrinol Metab. 2017;102(3):709-757.

8. de Onis M. WHO Child Growth Standards based on length/height, weight and age. Acta Paediatrica. 2007;95:76-85. 
9. Caprio S, Perry R, Kursawe R. Adolescent Obesity and Insulin Resistance: Roles of Ectopic Fat Accumulation and Adipose Inflammation. Gastroenterology. 2017;152(7):1638-1646.

10. Skinner AC, Perrin EM, Moss LA, Skelton JA. Cardiometabolic Risks and Severity of Obesity in Children and Young Adults. N Engl J Med. 2015;373(14):1307-1317.

11. CDC. Prevalence of Overweight and Obesity Among Children and Adolescents Aged 2 to 19 Years: United States, 1963- 1965 Through 2013-2014. http://www.cdc.gov/nchs/data/hestat/obesity child 13 14/obesity child 13 14.htm. Accessed Sep 2, 2017. 2016.

12. Ogden CL, Carroll MD, Lawman HG, et al. Trends in Obesity Prevalence Among Children and Adolescents in the United States, 1988-1994 Through 2013-2014. Jama. 2016;315(21):2292-2299.

13. Robertson W, Murphy M, Johnson R. Evidence base for the prevention and management of child obesity. Paediatrics and Child Health. 2016;26(5):212-218.

14. de Onis M. Preventing childhood overweight and obesity. J Pediatr (Rio J). 2015;91(2):105-107.

15. Weiss R, Kaufman FR. Metabolic Complications of Childhood Obesity. Identifying and mitigating the risk. 2008;31(Supplement 2):S310-S316.

16. Kelishadi R, Cook SR, Motlagh ME, et al. Metabolically obese normal weight and phenotypically obese metabolically normal youths: the CASPIAN Study. Journal of the American Dietetic Association. 2008;108(1):82-90. 
17. Maffetone PB, Rivera-Dominguez I, Laursen PB. Overfat Adults and Children in Developed Countries: The Public Health Importance of Identifying Excess Body Fat. Front Public Health. 2017;5(190):190.

18. Maffetone PB, Laursen PB. The Prevalence of Overfat Adults and Children in the US. Frontiers in Public Health. 2017;5(290).

19. Sims EA. Are there persons who are obese, but metabolically healthy? Metabolism: clinical and experimental. 2001;50(12):1499-1504.

20. Bluher S, Schwarz P. Metabolically healthy obesity from childhood to adulthood Does weight status alone matter? Metabolism: clinical and experimental. 2014;63(9):1084-1092.

21. Boonchaya-anant P, Apovian CM. Metabolically healthy obesity--does it exist? Current atherosclerosis reports. 2014;16(10):441.

22. Hinnouho GM, Czernichow S, Dugravot A, et al. Metabolically healthy obesity and the risk of cardiovascular disease and type 2 diabetes: the Whitehall II cohort study. Eur Heart J. 2015;36(9):551-559.

23. Goncalves CG, Glade MJ, Meguid MM. Metabolically healthy obese individuals: Key protective factors. Nutrition. 2016;32(1):14-20.

24. Schwartz MW, Seeley RJ, Zeltser LM, et al. Obesity Pathogenesis: An Endocrine Society Scientific Statement. Endocr Rev. 2017;38(4):267-296.

25. Farias MM, Cuevas AM, Rodriguez F. Set-point theory and obesity. Metabolic syndrome and related disorders. 2011;9(2):85-89.

26. Ahima RS. Revisiting leptin's role in obesity and weight loss. J Clin Invest. 2008;118(7):2380-2383. 
27. Korner J, Aronne LJ. The emerging science of body weight regulation and its impact on obesity treatment. J Clin Invest. 2003;111(5):565-570.

28. Kieffer TJ, Habener JF. The adipoinsular axis: effects of leptin on pancreatic betacells. Am J Physiol Endocrinol Metab. 2000;278(1):E1-E14.

29. Hao Z, Mumphrey MB, Townsend RL, et al. Body Composition, Food Intake, and Energy Expenditure in a Murine Model of Roux-en-Y Gastric Bypass Surgery. Obesity surgery. 2016;26(9):2173-2182.

30. Rosenbaum M, Sy M, Pavlovich K, Leibel RL, Hirsch J. Leptin reverses weight lossinduced changes in regional neural activity responses to visual food stimuli. J Clin Invest. 2008;118(7):2583-2591.

31. Rosenbaum M, Goldsmith R, Bloomfield D, et al. Low-dose leptin reverses skeletal muscle, autonomic, and neuroendocrine adaptations to maintenance of reduced weight. J Clin Invest. 2005;115(12):3579-3586.

32. Yu YH. Making sense of metabolic obesity and hedonic obesity. Journal of diabetes. 2017;9(7):656-666.

33. Huang JS, Barlow SE, Quiros-Tejeira RE, et al. Childhood obesity for pediatric gastroenterologists. Journal of pediatric gastroenterology and nutrition. 2013;56(1):99-109.

34. Camilleri M, Malhi H, Acosta A. Gastrointestinal Complications of Obesity. Gastroenterology. 2017;152(7):1656-1670.

35. Barshop NJ, Capparelli EV, Sirlin CB, Schwimmer JB, Lavine JE. Acetaminophen pharmacokinetics in children with nonalcoholic fatty liver disease. Journal of pediatric gastroenterology and nutrition. 2011;52(2):198-202. 
36. Ohaeri JU, Akanji AO. Metabolic syndrome in severe mental disorders. Metabolic syndrome and related disorders. 2011;9(2):91-98.

37. Massetti GM, Dietz WH, Richardson LC. Excessive Weight Gain, Obesity, and Cancer: Opportunities for Clinical Intervention. Jama. 2017.

38. Steele CB, Thomas CC, Henley SJ, et al. Vital Signs: Trends in Incidence of Cancers Associated with Overweight and Obesity - United States, 2005-2014. MMWR Morb Mortal Wkly Rep. 2017;66(39):1052-1058.

39. Domecq JP, Prutsky G, Leppin A, et al. Clinical review: Drugs commonly associated with weight change: a systematic review and meta-analysis. J Clin Endocrinol Metab. 2015;100(2):363-370.

40. Frattarelli DA, Galinkin JL, Green TP, et al. Off-label use of drugs in children. Pediatrics. 2014;133(3):563-567.

41. Macfarlane DP, Forbes S, Walker BR. Glucocorticoids and fatty acid metabolism in humans: fuelling fat redistribution in the metabolic syndrome. The Journal of endocrinology. 2008;197(2):189-204.

42. Fardet L, Feve B. Systemic glucocorticoid therapy: a review of its metabolic and cardiovascular adverse events. Drugs. 2014;74(15):1731-1745.

43. Huscher D, Thiele K, Gromnica-Ihle E, et al. Dose-related patterns of glucocorticoidinduced side effects. Annals of the rheumatic diseases. 2009;68(7):1119-1124.

44. Prednisone delayed release tablets. Rayos prescribing Information. 2012.

45. Robertson KJ, Schoenle E, Gucev Z, Mordhorst L, Gall MA, Ludvigsson J. Insulin detemir compared with NPH insulin in children and adolescents with Type 1 diabetes. Diabet Med. 2007;24(1):27-34. 
46. Thalange N, Bereket A, Larsen J, Hiort LC, Peterkova V. Insulin analogues in children with Type 1 diabetes: a 52-week randomized clinical trial. Diabet Med. 2013;30(2):216-225.

47. Zachariah S, Sheldon B, Shojaee-Moradie F, et al. Insulin detemir reduces weight gain as a result of reduced food intake in patients with type 1 diabetes. Diabetes Care. 2011;34(7):1487-1491.

48. Russell-Jones D, Danne T, Hermansen K, et al. Weight-sparing effect of insulin detemir: a consequence of central nervous system-mediated reduced energy intake? Diabetes, obesity \& metabolism. 2015;17(10):919-927.

49. Narasimhan S, Weinstock RS. Youth-onset type 2 diabetes mellitus: lessons learned from the TODAY study. Mayo Clinic proceedings. 2014;89(6):806-816.

50. Zeitler P, Hirst K, Copeland KC, et al. HbA1c After a Short Period of Monotherapy With Metformin Identifies Durable Glycemic Control Among Adolescents With Type 2 Diabetes. Diabetes Care. 2015;38(12):2285-2292.

51. Niswender K. Diabetes and obesity: therapeutic targeting and risk reduction - a complex interplay. Diabetes, obesity \& metabolism. 2010;12(4):267-287.

52. Kapur S, Marques TR. Dopamine, Striatum, Antipsychotics, and Questions About Weight Gain. JAMA psychiatry. 2016;73(2):107-108.

53. Reekie J, Hosking SP, Prakash C, Kao KT, Juonala M, Sabin MA. The effect of antidepressants and antipsychotics on weight gain in children and adolescents. Obesity reviews : an official journal of the International Association for the Study of Obesity. 2015;16(7):566-580. 
54. Varley CK, McClellan J. Implications of marked weight gain associated with atypical antipsychotic medications in children and adolescents. Jama. 2009;302(16):18111812.

55. Correll CU, Manu P, Olshanskiy V, Napolitano B, Kane JM, Malhotra AK. Cardiometabolic risk of second-generation antipsychotic medications during firsttime use in children and adolescents. Jama. 2009;302(16):1765-1773.

56. Correll CU. Antipsychotic use in children and adolescents: minimizing adverse effects to maximize outcomes. J Am Acad Child Adolesc Psychiatry. 2008;47(1):920.

57. Correll CU, Carlson HE. Endocrine and metabolic adverse effects of psychotropic medications in children and adolescents. J Am Acad Child Adolesc Psychiatry. 2006;45(7):771-791 .

58. Anagnostou E, Aman MG, Handen BL, et al. Metformin for Treatment of Overweight Induced by Atypical Antipsychotic Medication in Young People With Autism Spectrum Disorder: A Randomized Clinical Trial. JAMA psychiatry. 2016;73(9):928937.

59. Asenapine sublingual tablets. Saphris prescribing information. 2017.

60. Lurasidone film coated tablets. Latuda prescribing information. . 2017.

61. Paliperidone extended release tablets. Invega prescribing information. 2017.

62. Calarge CA, Mills JA, Janz KF, Burns TL, Coryell WH, Zemel BS. Body Composition in Adolescents During Treatment With Selective Serotonin Reuptake Inhibitors. Pediatrics. 2017;140(1). 
63. Merry SN, Hetrick SE, Stasiak K. Effectiveness and Safety of Antidepressants for Children and Adolescents: Implications for Clinical Practice. JAMA psychiatry. 2017;74(10):985-986.

64. Ben-Menachem E. Weight issues for people with epilepsy--a review. Epilepsia. 2007;48 Suppl 9:42-45.

65. Brodie MJ. Tolerability and Safety of Commonly Used Antiepileptic Drugs in Adolescents and Adults: A Clinician's Overview. CNS Drugs. 2017;31(2):135-147.

66. Torgerson JS, Hauptman J, Boldrin MN, Sjostrom L. XENical in the prevention of diabetes in obese subjects (XENDOS) study: a randomized study of orlistat as an adjunct to lifestyle changes for the prevention of type 2 diabetes in obese patients. Diabetes Care. 2004;27(1):155-161.

67. Diabetes Prevention Program Research G. The 10-year cost-effectiveness of lifestyle intervention or metformin for diabetes prevention: an intent-to-treat analysis of the DPP/DPPOS. Diabetes Care. 2012;35(4):723-730.

68. Freemark M, Bursey D. The effects of metformin on body mass index and glucose tolerance in obese adolescents with fasting hyperinsulinemia and a family history of type 2 diabetes. Pediatrics. 2001;107(4):E55.

69. Chanoine JP, Hampl S, Jensen C, Boldrin M, Hauptman J. Effect of orlistat on weight and body composition in obese adolescents: a randomized controlled trial. Jama. 2005;293(23):2873-2883.

70. Zhi J, Moore R, Kanitra L, Mulligan TE. Effects of orlistat, a lipase inhibitor, on the pharmacokinetics of three highly lipophilic drugs (amiodarone, fluoxetine, and simvastatin) in healthy volunteers. J Clin Pharmacol. 2003;43(4):428-435. 
71. Viner RM, Hsia Y, Neubert A, Wong ICK. Rise in antiobesity drug prescribing for children and adolescents in the UK: a population-based study. British Journal of Clinical Pharmacology. 2009;68(6):844-851.

72. Yanovski JA, Krakoff J, Salaita CG, et al. Effects of metformin on body weight and body composition in obese insulin-resistant children: a randomized clinical trial. Diabetes. 2011;60(2):477-485.

73. Kendall D, Vail A, Amin R, et al. Metformin in obese children and adolescents: the MOCA trial. J Clin Endocrinol Metab. 2013;98(1):322-329.

74. Wilson DM, Abrams SH, Aye $\mathrm{T}$, et al. Metformin extended release treatment of adolescent obesity: a 48-week randomized, double-blind, placebo-controlled trial with 48-week follow-up. Arch Pediatr Adolesc Med. 2010;164(2):116-123.

75. Rena G, Hardie DG, Pearson ER. The mechanisms of action of metformin. Diabetologia. 2017;60(9):1577-1585.

76. Wagner DJ, Hu T, Wang J. Polyspecific organic cation transporters and their impact on drug intracellular levels and pharmacodynamics. Pharmacol Res. 2016;111(Supplement C):237-246.

77. Sam WJ, Roza O, Hon YY, et al. Effects of SLC22A1 Polymorphisms on MetforminInduced Reductions in Adiposity and Metformin Pharmacokinetics in Obese Children With Insulin Resistance. J Clin Pharmacol. 2017;57(2):219-229.

78. Nathan BM, Rudser KD, Abuzzahab MJ, et al. Predictors of weight-loss response with glucagon-like peptide-1 receptor agonist treatment among adolescents with severe obesity. Clin Obes. 2016;6(1):73-78. 
79. Pi-Sunyer X, Astrup A, Fujioka K, et al. A Randomized, Controlled Trial of $3.0 \mathrm{mg}$ of Liraglutide in Weight Management. New England Journal of Medicine. 2015;373(1):11-22.

80. Danne T, Biester T, Kapitzke K, et al. Liraglutide in an Adolescent Population with Obesity: A Randomized, Double-Blind, Placebo-Controlled 5-Week Trial to Assess Safety, Tolerability, and Pharmacokinetics of Liraglutide in Adolescents Aged 12-17 Years. J Pediatr. 2017;181(Supplement C):146-153 e143.

81. Fox CK, Marlatt KL, Rudser KD, Kelly AS. Topiramate for weight reduction in adolescents with severe obesity. Clinical pediatrics. 2015;54(1):19-24.

82. Dai D, Feinstein JA, Morrison W, Zuppa AF, Feudtner C. Epidemiology of Polypharmacy and Potential Drug-Drug Interactions Among Pediatric Patients in ICUs of U.S. Children's Hospitals. Pediatric critical care medicine : a journal of the Society of Critical Care Medicine and the World Federation of Pediatric Intensive and Critical Care Societies. 2016;17(5):e218-228.

83. Vaughns JD, Ziesenitz VC, van den Anker JN. Clinical Pharmacology of Frequently Used Intravenous Drugs During Bariatric Surgery in Adolescents. Current pharmaceutical design. 2015;21(39):5650-5659.

84. Abernethy DR, Divoll M, Greenblatt DJ, Ameer B. Obesity, sex, and acetaminophen disposition. Clin Pharmacol Ther. 1982;31(6):783-790.

85. Lee WH, Kramer WG, Granville GE. The effect of obesity on acetaminophen pharmacokinetics in man. J Clin Pharmacol. 1981;21(7):284-287. 
86. van Rongen A, Valitalo PA, Peeters MY, et al. Morbidly Obese Patients Exhibit Increased CYP2E1-Mediated Oxidation of Acetaminophen. Clin Pharmacokinet. 2016;55(7):833-847.

87. Natale S, Bradley J, Nguyen WH, et al. Pediatric Obesity: Pharmacokinetic Alterations and Effects on Antimicrobial Dosing. Pharmacotherapy. 2017;37(3):361378.

88. Sampson M, Cohen-Wolkowiez M, Benjamin D, Jr., Capparelli E, Watt K. Pharmacokinetics of Antimicrobials in Obese Children. GaBI journal. 2013;2(2):7681.

89. Janson B, Thursky K. Dosing of antibiotics in obesity. Current opinion in infectious diseases. 2012;25(6):634-649.

90. Wurtz R, Itokazu G, Rodvold K. Antimicrobial dosing in obese patients. Clinical infectious diseases : an official publication of the Infectious Diseases Society of America. 1997;25(1):112-118.

91. Kendrick JG, Carr RR, Ensom MH. Pediatric Obesity: Pharmacokinetics and Implications for Drug Dosing. Clinical therapeutics. 2015;37(9):1897-1923.

92. Rowe S, Siegel D, Benjamin DK, Jr., Best Pharmaceuticals for Children Act Pediatric Trials Network Administrative Core C. Gaps in Drug Dosing for Obese Children: A Systematic Review of Commonly Prescribed Emergency Care Medications. Clinical therapeutics. 2015;37(9):1924-1932.

93. Shah M, Hurt RT, Mundi MS. Phenotypes of Obesity: How it Impacts Management. Curr Gastroenterol Rep. 2017;19(11):55. 
94. van Rongen A, Brill MJE, Vaughns JD, et al. Higher Midazolam Clearance in Obese Adolescents Compared with Morbidly Obese Adults. Clin Pharmacokinet. 2017. 